\title{
Viscoelastic Characterization of Long-Eared Owl Flight Feather Shaft and the Damping Ability Analysis
}

\author{
Jia-li Gao, ${ }^{1}$ Jin-kui Chu, ${ }^{1}$ Le Guan, ${ }^{1}$ Hai-xin Shang, ${ }^{1}$ and Zhen-kun Lei ${ }^{2}$ \\ ${ }^{1}$ Key Laboratory for Precision \& Non-Traditional Machining of Ministry of Education, Dalian University of Technology, \\ Dalian 116024, China \\ ${ }^{2}$ State Key Laboratory of Structural Analysis for Industrial Equipment, Dalian University of Technology, Dalian 116024, China \\ Correspondence should be addressed to Jin-kui Chu; chujk@dlut.edu.cn
}

Received 25 May 2014; Revised 28 July 2014; Accepted 29 July 2014; Published 28 August 2014

Academic Editor: Mickaël Lallart

Copyright (C) 2014 Jia-li Gao et al. This is an open access article distributed under the Creative Commons Attribution License, which permits unrestricted use, distribution, and reproduction in any medium, provided the original work is properly cited.

\begin{abstract}
Flight feather shaft of long-eared owl is characterized by a three-parameter model for linear viscoelastic solids to reveal its damping ability. Uniaxial tensile tests of the long-eared owl, pigeon, and golden eagle flight feather shaft specimens were carried out based on Instron 3345 single column material testing system, respectively, and viscoelastic response of their stress and strain was described by the standard linear solid model. Parameter fitting result obtained from the tensile tests shows that there is no significant difference in instantaneous elastic modulus for the three birds' feather shafts, but the owl shaft has the highest viscosity, implying more obvious viscoelastic performance. Dynamic mechanical property was characterized based on the tensile testing results. Loss factor (tan $\delta$ ) of the owl flight feather shaft was calculated to be $1.609 \pm 0.238$, far greater than those of the pigeon $(0.896 \pm 0.082)$ and golden eagle $(1.087 \pm 0.074)$. It is concluded that the long-eared owl flight feather has more outstanding damping ability compared to the pigeon and golden eagle flight feather shaft. Consequently, the long-eared owl flight feathers can dissipate the vibration energy more effectively during the flying process based on the principle of damping mechanism, for the purpose of vibration attenuation and structure radiated noise reduction.
\end{abstract}

\section{Introduction}

Noise pollution is a major problem in the main urban traffic area recently, especially the airport neighborhoods, adversely affecting the lives of millions of people and animals. Evolving a silent flight, most genera of owls are not audible to man and, more importantly, to their prey. How flight noise can be efficiently reduced may be learned from owls.

Since more than a century ago, the research regarding the mechanisms that enable the nearly silent flight of owls has remained an interesting field for theoretical and experimental research. Mascha and Graham discovered and innovatively concluded three mechanisms of the owl feathers that supposedly enabled the silent flight: (1) comb-like structure at the leading edge of the wings, (2) long and soft fringes at the trailing edge, and (3) soft downy upper surface of the feathers $[1,2]$. These three mechanisms have been gradually identified [3-6] and accepted by the bionic researchers [713]. The reported biological studies of the noiselessly flying owls show that owl wing feather has significantly long soft feather material and unique morphological structure, which are different from other bird feathers.

However, material research of the owl feathers is still not fully carried out and is away from material biomimetic synthesis so that bionic studies about the owl feather are almost all based on the outline edges. Though Bachmann et al. experimentally measured the flexural stiffness of the owl primary feather shaft cortex [14], a more comprehensive mechanical characterization of owl feather is necessary. In addition, its comparison with other bird feathers is still insufficient.

Stiff, strong, but lightweight shaft is the key force bearing structure in an owl feather for its gliding flight. Interacting with the flow field during flight, feather shafts are subjected 
to bend mainly under aerodynamic loads $[15,16]$. Because relative movement caused by the feather vibration and friction during flight would lead to the radiation noise [17], amplitude stability of the feather shaft directly affects the vibration of the whole feather structure and noise radiation. Damping factor as an important physical parameter can be used to characterize the vibration attenuation capability of the owl feather shaft and compared with other bird's shafts. Consequently, on account of the assumption that the investigation of biomimetic materials inspired by the quiet flying owls might lighten a new way to obtain more excellent muffling performance in addition to the morphological bionic design, we present our study on the viscoelastic characterization of owl flight feather materials and the analysis of their vibration damping ability, so as to provide further insight into the owl's silent flight mechanism and bionic design in our further research in view of material acoustics $[18,19]$.

The research presented in this paper focuses on the viscoelastic characterization of flight feather shaft of longeared owl (Asio otus, Strigiformes), pigeon (Columba livia, Columbiformes), and golden eagle (Aquila chrysaetos, Falconiformes) to reveal the damping ability of their dynamic feathers on wings. Here, long-eared owl is the main research object with noise reduction function [20]. Its flight generally consists of an initial flapping phase followed by a gliding phase and a short flapping during touchdown [21]. Pigeon is a typical flapping flight bird which differs from the long-eared owl and has a smaller size than the owl. Golden eagle involves the same flight style as the long-eared owl and its size is larger than the long-eared owl. Both of the pigeon and golden eagle do not have the silent flight ability. The aim is to compare the viscoelastic performance of owl feather with other noisy flying birds without the impact of flying style and bird size on noise generation.

Feather shaft can be regarded as a slender beam, so the shear effect on its bending deformation can be ignored. Hence, normal stress accounts for the bending deformation of feather shaft. Therefore, uniaxial tensile tests of the three types of the prepared flight feather shaft specimens were conducted on Instron 3345 single column materials testing system to investigate the relationship of strain and normal stress of the shafts at first. Then, viscoelasticity of feather shaft material was studied employing the standard linear viscoelastic solid model. And then, based on the viscoelastic material parameters obtained from the tensile testing results, dynamic mechanical property at a constant temperature was analyzed. And $\tan \delta$ of the long-eared owl, pigeon, and golden eagle flight feather shaft was calculated for a damping ability comparison between the three types of flight feathers. Relationship between damping ability and vibration attenuation for owl feather shaft was briefly discussed at last.

\section{Materials and Methods}

2.1. Feather Material and Anatomy of Feather Shafts. Flight feathers of long-eared owl, pigeon, and golden eagle were obtained from Dalian Forest Zoo (Liaoning, China). It is noted that feathers are collected with no harm to the birds' life, complying with the Animal Protection Act. Barbs on both sides of the feather shaft were removed by the scalpel blade. Trimmed feather shafts were rinsed twice with deionized water for 30 minutes and naturally dried on clean filter paper at room temperature. All the specimens were cleaned and prepared in the cleanroom of key laboratory for precision and nontraditional machining of Ministry of Education, Dalian University of Technology.

The tested specimens were selected from the feather shafts according to the following rules:

(a) the same relative position that the testing samples are selected in the same proportion position on flight feather shafts of the long-eared owl, pigeon, and golden eagle,

(b) the same aspect ratio that the ratio of the shaft length to maximum cross-sectional diameter is unified for all the testing parts: one end of the testing section is marked according to the selection method (a) at first, and its maximum diameter size is measured by a spiral micrometer subsequently. The other end is located away from the marked position with a gauge length of fifteen times the maximum diameter.

After marking the testing portion in accordance with the above methods, specimen including the testing section and sufficient extension parts on its two sides for clamping is cut out, as is shown in Figure 1. Before the test is started, reinforcement of the clamping parts is necessary in order to avoid damage of the specimen caused by gripping. Two extension parts were inserted in flat boxes which are full of liquid epoxy structural adhesive (WD3003) with high bonding strength and excellent performance to resist the compression. Clamping structures were obtained when the liquid adhesive was solidified into a block after 24 hours. Meanwhile, thin aluminium sheets with strong ductility were pasted on gripping interface of the adhesive to prevent contamination of the experimental equipment.

\subsection{Experimental Instrument and Procedures. Instron 3345} single column materials testing system (Instron Corporation, the United States) is used for uniaxial tensile test, as is shown in Figure 2. The whole system is equipped with a $5 \mathrm{kN}$ load cell, a displacement sensor, a speed-controlled guide screw driving device (range: $0.005 \mathrm{~mm} / \mathrm{min}$ to $1000 \mathrm{~mm} / \mathrm{min}$, accuracy: $\pm 5 \%$ of the point value), and wedge clamps with the gripping force of $50 \mathrm{kN}$ that one clamp is connected to the load cell while the other is secured to the machine. Both the load accuracy and the strain accuracy are $\pm 5 \%$ of indicated value for the materials testing system.

During the experiments, the material testing system recorded the stretching force every 0.1 seconds and the oneto-one sampling time until the testing specimen was fractured. Digital image correlation (DIC) was applied to analyze the deformation mechanisms [22]. Firstly, two high contrast markers were spotted on the lateral wall of the specimen. Secondly, high-speed camera was used to obtain charge coupled device (CCD) images before and after the tensile deformation in the experimental process. The corresponding 


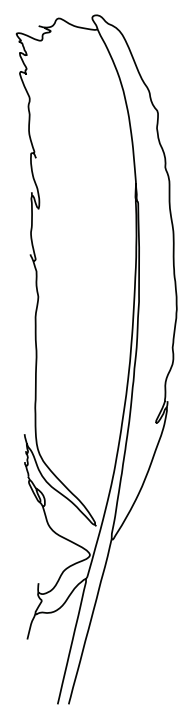

(a)

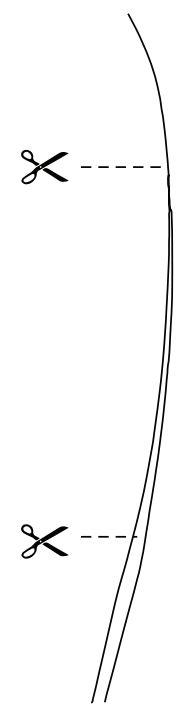

(b)

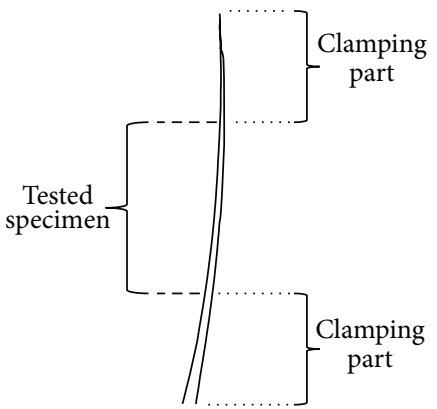

(c)

FIGURE 1: Schematic drawings of flight feather and the sampling of tensile tested specimen. (a) Complete feather. (b) Feather shaft after removing the vane with scissors. (c) Tested specimen with extension parts on its two sides for clamping.

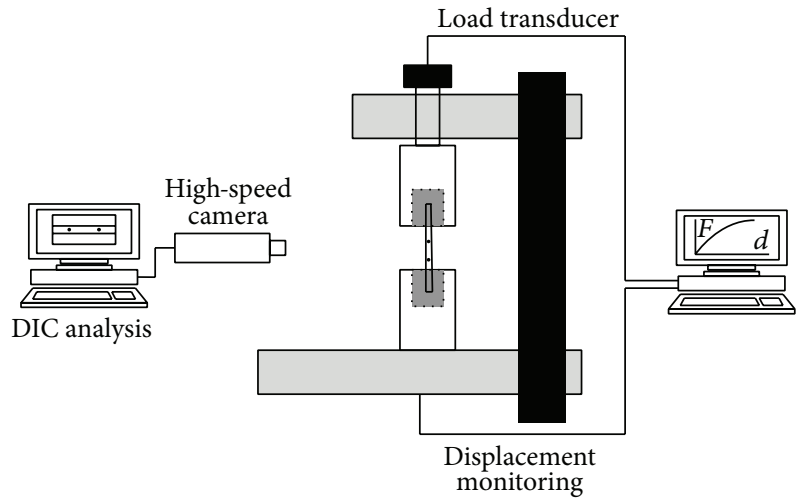

FIGURE 2: Schematic of the tensile test. The test specimen (feather shaft) was center-mounted onto the wedge clamps. Tensile force $(F)$ was measured while the specimen was pulled at a constant rate of $0.5 \mathrm{~mm} / \mathrm{min}$. Tensile process was recorded by a high speed camera and the strain of the specimen was calculated from DIC analysis of the CCD images. DIC analysis was applied with the purpose of excluding the influence caused by the sample debonding, clamping, and the measurement error of sample's initial length.

elongated deformation of the specimen was computed from the extremum of correlation coefficient by the correlation calculation of the CCD images finally. CCD images of each loading in the whole test were recorded and the real strain at each observing time was calculated. All samples were tested at a constant room temperature of $22^{\circ} \mathrm{C}$ and humidity of $55 \%$.

\section{Results}

3.1. Morphological Characterization of the Feather Shaft Cross Section. Intersecting surface of the long-eared owl, pigeon, and golden eagle flight feather shaft was morphologically

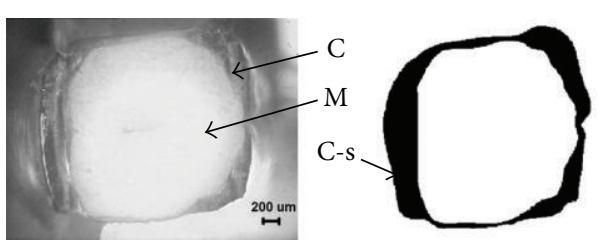

(a)

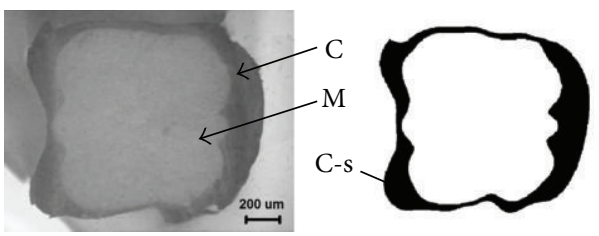

(b)
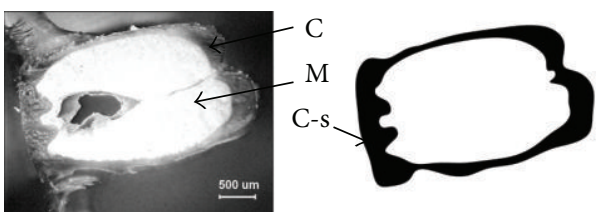

(c)

FIGURE 3: Flight feather shaft cross-section and scanning crosssection of the cortex. (a) Cross-section of long-eared owl feather. (b) Cross-section of pigeon feather. (c) Cross-section of golden eagle feather. C: Cortex; M: Medulla; C-s: Cross-section of the cortex.

characterized, as shown in Figure 3. The general morphology of the rachis is very similar in the three species. All shafts have an approximate shape of a box girder; each shaft consists of a solid compact shell (cortex) and is filled by the medulla, which contains air-filled keratinized epithelial cells with an average diameter of $10-20 \mu \mathrm{m}$ [23]. Noteworthy is the 
asymmetry of the cross section of a considerable part of the flight feather shaft.

According to the reported literatures, the feather shaft gives rise to X-ray diffraction patterns of great complexity that they have been described as the most complex known for the naturally occurring fibrous substances. And further study suggests that $\beta$-keratin structure is proposed to account for the principal features of the X-ray pattern and for some physical properties of the feather shaft. Cortex is composed of two different $\beta$-keratin fiber layers where the inner layer fiber direction is along the shaft and the outer keratin fiber direction is circumferential. Due to its high hardness and great flexibility, cortex bears most bending stresses in the flight process. The compact keratin was found to be approximately 100 times stiffer than the medullary foam [24], consistent with an earlier finding of Purslow and Vincent that the medullary foam and transverse septa contribute only $16 \%$ to the overall bending stiffness of the feather shaft [25]. Because the medullary foam appears to play a minor structural role, calculation of the medulla structure is negligible in this study and our research is focused on the cortex for all flight feather shafts.

Cross-sectional area of the fractured position of the feather shaft cortex was calculated using image extraction method. Cross-sectional area of the tested long-eared owl specimen is $1.360 \pm 0.103 \mathrm{~mm}^{2}$, cross-sectional area of pigeon specimen is $0.308 \pm 0.024 \mathrm{~mm}^{2}$, and cross-sectional area of golden eagle specimen is $1.511 \pm 0.273 \mathrm{~mm}^{2}$.

3.2. Mechanical Behavior of the Flight Feather Shafts. Figure 4 gives a description of the flight feather shaft of long-eared owl, pigeon, and golden eagle specimens before and after experiments, respectively. Fractured positions are mostly located near to the thin end of the gauge length for shaft specimens. The fracture mode of the shafts is ductile fracture. Influence caused by the sample fixation, clamping, and adhesive debonding can be avoided since tensile strain is calculated by DIC analysis.

Figure 5 presents typical stress-strain curves of longeared owl's and pigeon's flight feather shaft. Stress $(\sigma)$ of the shaft relative to the cross-sectional area $(A)$ is given by the formula $\sigma=F / A$ where $F$ is the tensile force. In addition, the formula for the strain $(\varepsilon)$ is also given as $\varepsilon=L^{\prime} / L$ where $L$ is the feather shaft's initial gauge length and $L^{\prime}$ is the drawn shaft length, respectively. Nonlinear stress-strain relationship is a common feature for all shafts in Figure 5. And it can also be found in Figure 5 that stress versus strain curve curvature of the owl shaft is larger than the other two curves.

\section{Discussions}

4.1. Viscoelastic Behavior and Model Analysis. The above uniaxial tensile test results reveal a nonlinear material behavior. Based on the reported achievements in relation to natural biological composite materials, asymmetrical geometry, viscoelastic property, and inhomogeneous density are inherent characteristics for feather shaft, leading to the nonlinear response between stress and strain during the loading process. In this study, the selected testing samples from longeared owl, pigeon, and golden eagle feather shaft were straight as far as possible in length direction and the cortex sustained most of the tensile stress. Therefore, asymmetrical geometry and inhomogeneous density of the material are not the determinate factors for nonlinear mechanical response. As a result, it is suggested that viscoelastic property might be the major factor causing the nonlinear stress-strain relationship of the feather shaft.

In consideration of the nature of the tested material and the applied testing method, we employed a three-parameter standard linear solid [26] to describe the nonlinear stressstrain relation of feather shaft. This nonlinear model is subject to uniaxial tensile test. The model consists of a single elastic element (spring) (elastic modulus $E_{1}$ ) and a Kelvin element with an elastic element (elastic modulus $E_{2}$ ) and a viscous element (dashpot) (coefficient of viscosity $\eta$ ) in parallel. The constitutive law in differential equation form for the standard linear solid model is

$$
\sigma+\frac{\eta}{E_{1}+E_{2}} \dot{\sigma}=\frac{E_{1} E_{2}}{E_{1}+E_{2}} \varepsilon+\frac{E_{1} \eta}{E_{1}+E_{2}} \dot{\varepsilon}
$$

Delay time $\left(\tau_{c}=\eta / E_{2}\right)$ and relaxation time $\left(\tau_{R}=\eta /\left(E_{1}+E_{2}\right)\right)$ are two important internal time parameters for viscoelastic material. And for the standard linear solid model, $\tau_{c}$ and $\tau_{R}$ only relate to material constants $\left(E_{1}, E_{2}, \eta\right)$.

In our test, shaft specimens were tested at a constant rate. Therefore, mechanical behavior was studied under the condition of constant strain ratio $(T)$. Stress response was investigated under the whole strain history. Strain expression is as follows:

$$
\varepsilon(t)=T t
$$

Employing Boltzmann's superposition method, constitutive equation of the stress response with a loading history of constant strain ratio is obtained:

$$
\begin{aligned}
\sigma(t) & =\int_{\tau=\infty}^{\tau=\infty} E(t-\tau) d \varepsilon(\tau)=E(t) * d \varepsilon(t) \\
& =\frac{E_{1} T}{E_{1}+E_{2}}\left[E_{2} t+\frac{E_{1} \eta}{E_{1}+E_{2}}\left(1-e^{-\left(\left(E_{1}+E_{2}\right) / \eta\right) t}\right)\right] .
\end{aligned}
$$

Dynamic behavior of viscoelastic materials is analyzed when alternating stress (4) with angular frequency $(\omega)$ is applied. Thus, strain response of the viscoelastic solid can be expressed in (5):

$$
\begin{gathered}
\sigma(t)=\sigma_{0} e^{i \omega t} \\
\varepsilon(t)=G(i \omega) \sigma(t)=G(i \omega) \sigma_{0} e^{i \omega t} .
\end{gathered}
$$

$G(i \omega)$ is defined as complex compliance, an elastic constant which is equal to the ratio of strain and stress. It changes only with frequency and is a characterization of dynamic 


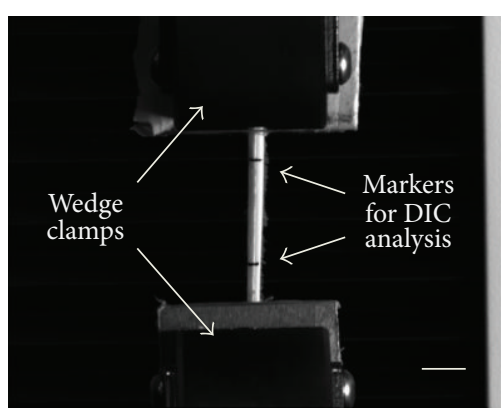

(a1)

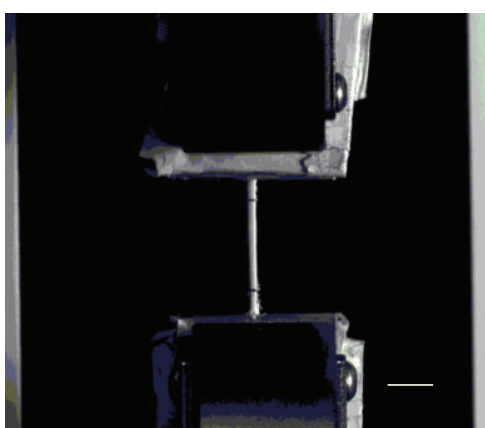

(b1)

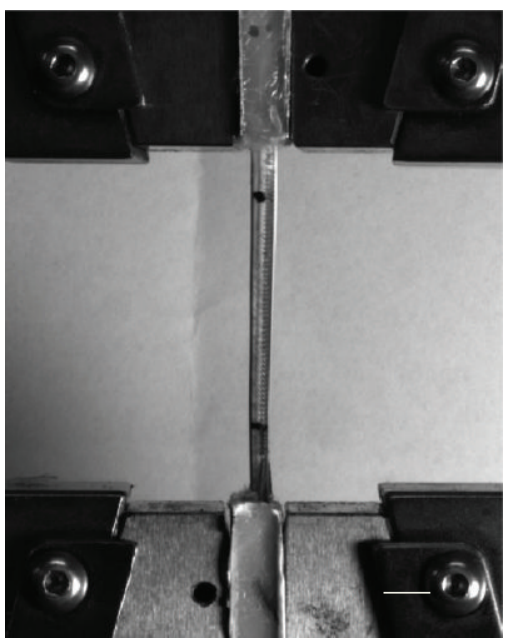

(c1)

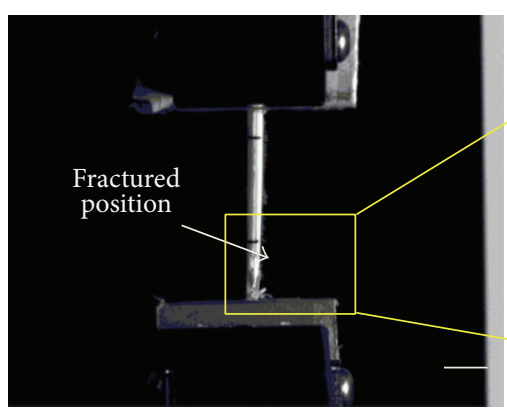

(a2)

(a)

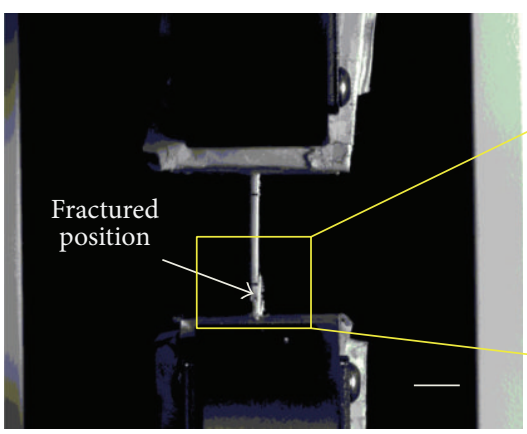

(b2)

(b)

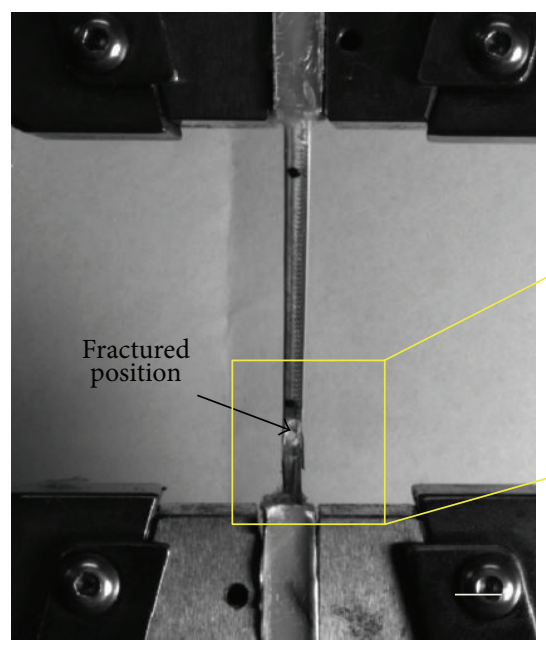

(c2)

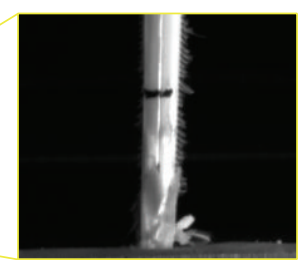

(a3)

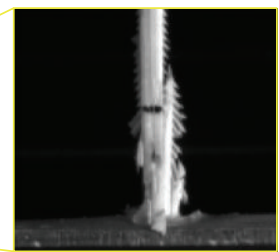

(b3)

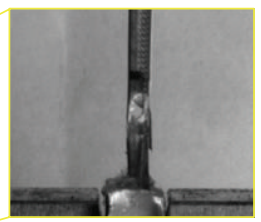

(c3)

(c)

FIGURE 4: Experimental specimens in the clamps, scale bar: $1 \mathrm{~mm}$. (a1) Fixed long-eared owl specimen before test. (a2) Breaking long-eared owl specimen after tensile process. (a3) Breaking point close-up of the owl specimen. (b1) Fixed pigeon specimen before test. (b2) Breaking pigeon specimen after tensile process. (b3) Breaking point close-up of the pigeon specimen. (c1) Fixed golden eagle specimen before test. (c2) Breaking golden eagle specimen after tensile process. (c3) Breaking point close-up of the golden eagle specimen.

mechanical properties of material in stable vibration. Complex compliance is used and is to be presented as follows:

$$
G(i \omega)=G_{1}(\omega)+i G_{2}(\omega)
$$

where $G_{1}$ and $G_{2}$ are identified as storage compliance and loss compliance, respectively. Equations (4) and (5) are put into
(1); thus, complex compliance $G(i \omega)$ of the standard linear solid model can be obtained in:

$$
\begin{aligned}
G(i \omega)= & \frac{E_{1}+E_{2}+\eta i \omega}{\left(E_{1} E_{2}\right)^{2}+\left(E_{1} \eta \omega\right)^{2}} \\
& \times\left[\left(E_{1}+E_{2}\right) E_{1} E_{2}+E_{1}(\eta \omega)^{2}-E_{1}^{2} \eta i \omega\right] .
\end{aligned}
$$




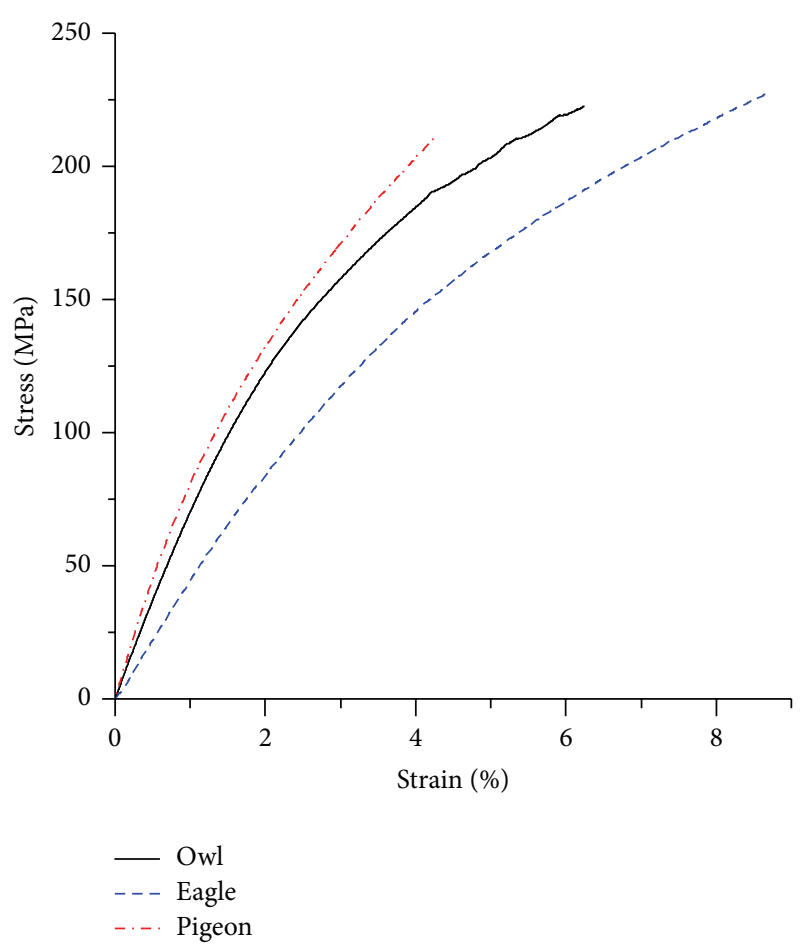

FIGURE 5: Relationships of the stress and strain for long-eared owl and pigeon shafts.

Viscoelastic parameters can be derived from the storage compliance and loss compliance. One parameter is the loss factor $(\tan \delta)$, where $\delta$ is an indication of the phase lag between the stress and strain. $\tan \delta$ represents the damping ability of the viscoelastic material and can be quantified by the ratio of the loss modulus to storage modulus:

$$
\begin{aligned}
\tan \delta & =-\frac{G_{2}(\omega)}{G_{1}(\omega)}=\frac{\left(\left(\eta / E_{2}\right)-\left(\eta /\left(E_{1}+E_{2}\right)\right)\right) \omega}{1+\left(\eta / E_{2}\right) \cdot\left(\eta /\left(E_{1}+E_{2}\right)\right) \omega^{2}} \\
& =\frac{\left(\tau_{c}-\tau_{R}\right)}{\sqrt{\tau_{c} \tau_{R}}} \cdot \frac{\omega \sqrt{\tau_{c} \tau_{R}}}{1+\tau_{c} \tau_{R} \omega^{2}} .
\end{aligned}
$$

From (8), it can be easily found that the maximum of $\tan \delta$ is obtained when $\sqrt{\tau_{c} \tau_{R}}=1$, and $\tan \delta$ is close to zero when $\omega$ approaches either zero or extremum $(\infty)$.

4.2. Determination of the Material Constants $\left(E_{1}, E_{2}, \eta\right)$. Least square method was employed to formulation regression for material constants prediction. The method of least squares is a standard approach to the approximate solution of overdetermined systems, mostly used in data fitting. The best fit in the least squares sense minimizes the sum of squared residuals, a residual being the difference between an observed value and the fitted value provided by a model. Least squares problems fall into two categories: linear or ordinary least squares and nonlinear least squares, depending on whether or not the residuals are linear in all unknowns.

In our test, the measured strain performs a linear enlargement with the increase of time. Relationship between the measured stress and testing time exhibits a nonlinear characteristic due to stress relaxation for the viscoelastic materials. SPSS statistical software was used following the principle of least square method for the linear fit of strain and loading time according to (2) firstly. Strain ratio $(T)$ is determined by the slope of the fitted straight line. And then non-linear fitting of the stress and loading time is conducted on the base of (3) for viscoelastic parameters $E_{1}, E_{2}, \eta$.

4.3. Fitted Material Parameters of Long-Eared Owl and Pigeon Flight Feather Shafts. Uniaxial tensile tests of the long-eared owl, pigeon, and golden eagle flight feather shaft specimens were performed and their fitted material parameters based on the three-parameter standard linear solid model were listed in Table 1. Five specimens were tested for each shaft sample group.

From Table 1, it can be found that the golden eagle feather shaft has the lowest instantaneous elastic modulus $\left(E_{1}\right)((7273 \pm 661) \mathrm{MPa})$ and that of the pigeon feather shaft $((8841 \pm 687) \mathrm{MPa})$ is the highest. $E_{1}$ of the owl feather shaft falls in between the pigeon and golden eagle shafts. The conclusion is in accordance with Worcester's investigation that, among species, larger birds have more flexible primaries than smaller birds [27]. However, material parameters of Kelvin element (elastic modulus $\left(E_{2}\right)$ of the spring and viscosity coefficient $(\eta)$ of the dashpot) have greater dissimilarities. $E_{2}$ of long-eared owl flight feather shaft was $(722 \pm 149) \mathrm{MPa}$, lower than the one of pigeon feathers $(2849 \pm 718) \mathrm{Mpa}$ and golden eagle feathers $(1315 \pm 182) \mathrm{Mpa} . \eta$ of long-eared owl feathers feather shaft was $(17.06 \pm 1.70) * 10^{5} \mathrm{Mpa} \cdot \mathrm{s}$, higher than that of pigeon feathers $(7.99 \pm 1.57) * 10^{5} \mathrm{Mpa} \cdot \mathrm{s}$ and golden eagle feathers $(11.00 \pm 1.20) * 10^{5} \mathrm{Mpa} \cdot \mathrm{s}$. This indicates that the long-eared owl flight feather shaft is more like a typical viscoelastic material, but the pigeon and golden eagle flight feather shafts are more like elastic materials with a lower viscosity. Thus, more internal energy dissipation would occur by long-eared owl flight feather due to its molecular friction during the deformation process on the basis of viscoelastic theory.

4.4. $\tan \delta$ of Long-Eared Owl and Pigeon Flight Feather Shafts. $\tan \delta$ of the tested long-eared owl, pigeon, and golden eagle flight feather shafts is calculated for damping ability characterization from the fitted material parameters of uniaxial tensile test results according to (8), when alternating stress is applied to the specimens at constant testing temperature.

Figure 6 illustrates the loss tangent $(\tan \delta)$ of the longeared owl, pigeon, and golden eagle flight feather shafts. And Table 2 lists their peak value of $\tan \delta$. It is found that $\tan \delta_{\max }$ of the long-eared owl flight feather shafts $(1.609 \pm 0.238)$ is higher than the pigeon feather shafts $(0.896 \pm 0.082)$ and golden eagle shafts $(1.087 \pm 0.074)$. In other words, damping ability of the long-eared owl flight feather is better than the pigeon and golden eagle feather. Therefore, long-eared owl flight feather would dissipate more energy from the wing vibration in the flying process. The owl feather can restrain the resonance response more effectively due to its large damping factor. Thus, high damping ability is concluded to 
TABLE 1: Fitting parameters based on the viscoelastic model from tensile tests for the long-eared owl, pigeon, and golden eagle flight feather shafts.

\begin{tabular}{|c|c|c|c|c|c|}
\hline Sample & $\begin{array}{c}\text { Cross-sectional } \\
\text { area } A\left(\mathrm{~mm}^{2}\right)\end{array}$ & $\begin{array}{c}\text { Gauge length } L \\
(\mathrm{~mm})\end{array}$ & $\begin{array}{c}\text { Elastic modulus } \\
E_{1}(\mathrm{MPa})\end{array}$ & $\begin{array}{c}\text { Elastic modulus } \\
E_{2}(\mathrm{MPa})\end{array}$ & $\begin{array}{c}\text { Coefficient of } \\
\text { viscosity } \eta(\mathrm{MPa} \cdot \mathrm{s})\end{array}$ \\
\hline owl1 & 1.432 & 38.94 & 8210 & 625 & 1620290 \\
\hline owl2 & 1.474 & 34.28 & 8989 & 554 & 1684931 \\
\hline owl3 & 1.279 & 29.54 & 8318 & 896 & 1966866 \\
\hline owl4 & 1.384 & 38.40 & 8760 & 860 & 1747912 \\
\hline owl5 & 1.230 & 34.16 & 9930 & 673 & 1510543 \\
\hline pigeonl & 0.280 & 19.84 & 10087 & 2411 & 597359 \\
\hline pigeon2 & 0.324 & 22.98 & 9590 & 2314 & 981513 \\
\hline pigeon3 & 0.330 & 21.32 & 11103 & 3851 & 836727 \\
\hline pigeon 4 & 0.282 & 21.08 & 10201 & 3373 & 680685 \\
\hline pigeon5 & 0.322 & 23.46 & 10404 & 2296 & 896814 \\
\hline eagle1 & 1.197 & 39.48 & 7023 & 1333 & 1047637 \\
\hline eagle2 & 1.784 & 42.14 & 6674 & 1073 & 1107921 \\
\hline eagle3 & 1.710 & 43.48 & 7255 & 1197 & 1265107 \\
\hline eagle4 & 1.624 & 37.86 & 8396 & 1455 & 938756 \\
\hline eagle5 & 1.242 & 38.97 & 7016 & 1515 & 1141217 \\
\hline
\end{tabular}

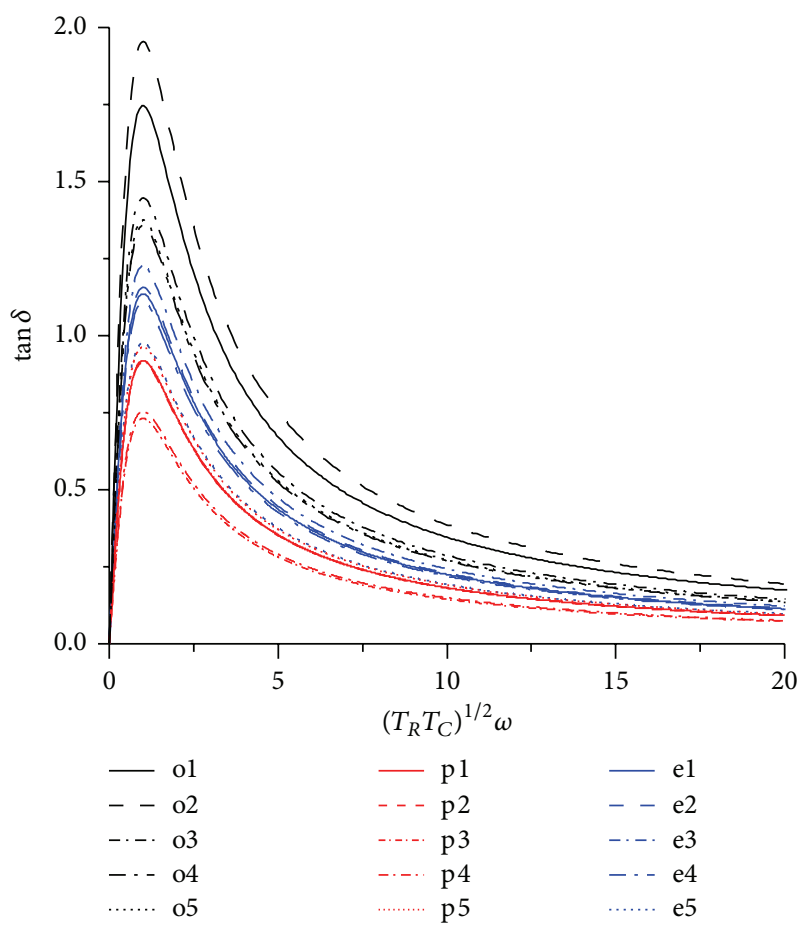

FIGURE 6: Internal friction spectrum of feather shaft specimens.

be an important factor to reduce or eliminate the mechanical vibration and free vibration of the owl feathers as soon as possible. As a result, noise caused by wing vibration and feather friction from the relative motion would effectively be suppressed by the long-eared owl.

\section{Conclusion}

Flight feather shafts of long-eared owl, pigeon, and golden eagle were mechanically characterized based on Instron 3345 single column material testing system at a constant loading rate. Nonlinear response of their stress and strain obtained from the uniaxial tensile tests was described by the standard linear solid model to reveal the viscoelastic characteristic of dynamic feathers on owls, pigeons, and golden eagle. The parameter fitting result of the three-parameter model indicates that the long-eared owl flight feather shaft is more like a typical viscoelastic material, but the pigeon and golden eagle flight feather shafts are more like elastic materials with a lower viscosity. Thus, more internal energy dissipation would occur by long-eared owl flight feather due to its molecular friction during the deformation process on the basis of viscoelastic theory.

In order to compare the damping ability of the three birds' feather shaft more intuitively, dynamic mechanical property at a constant temperature was characterized based on the parameter fitting result of the three-parameter model. Loss factor $(\tan \delta)$ of the long-eared owl flight feather shaft was calculated to be $1.609 \pm 0.238$, far greater than those of the pigeon $(0.896 \pm 0.082)$ and golden eagle $(1.087 \pm 0.074)$. It is concluded that the long-eared owl flight feather has more outstanding damping ability compared to pigeon and golden eagle flight feather shaft. Consequently, the flight feathers of the long-eared owl can dissipate the vibration energy more effectively in the form of heat during the flying process based on the principle of damping mechanism. Because relative movement caused by the feather vibration and friction would lead to the radiation noise, large damping factor which is efficient in rapid vibration attenuation could be assumed to reduce the flight noise greatly for the flight feather shaft. 
TABle 2: Peak value of the loss tangent $(\tan \delta)$ of the feather shaft specimens.

\begin{tabular}{lccccc}
\hline Owl specimen & $\tan \delta_{\max }$ & Pigeon specimen & $\tan \delta_{\max }$ & Eagle specimen & 1 \\
\hline 1 & 1.746 & 1 & 0.919 & 2 & 1.052 \\
2 & 1.954 & 2 & 0.914 & 3 & 1.157 \\
3 & 1.447 & 3 & 0.932 & 4 & 1.140 \\
4 & 1.522 & 4 & 0.754 & 5 & 1.109 \\
5 & 1.376 & 5 & 0.963 & 0.976 \\
\hline
\end{tabular}

In this paper, noise reduction mechanism for long-eared owl was investigated from the viscoelastic viewpoint of flight feather materials. We hope the conclusion would offer a new perspective for the research in this area.

\section{Conflict of Interests}

The authors declare that there is no conflict of interests regarding the publication of this paper.

\section{Acknowledgments}

The authors would like to thank Dalian Forest Zoo for providing the feather samples of long-eared owl, pigeon, and golden eagle. The authors also appreciate the constructive suggestions of Dr. Xuanping Wang from the Institute of Advanced Manufacturing Technology, Dalian University of Technology, at various stages of the work. Financial support for this research was provided by the National Basic Research Program of China (Grants 2011CB302101 and 2011CB302105) and the National Natural Science Foundation of China (Grant no. 51305057).

\section{References}

[1] R. Graham, "The silent flight of owls," Journal of the Royal Aeronautical Society, vol. 38, pp. 837-843, 1934.

[2] E. Mascha, "Über die Schwungfedern," Zeitschrift für Wissenschaftliche Zoologie, vol. 77, pp. 606-651, 1903.

[3] T. Bachmann, "The silent flight of owls," Integrative and Comparative Biology, vol. 52, article E9, 2012.

[4] T. Bachmann, H. Wagner, and C. Tropea, "Inner vane fringes of barn owl feathers reconsidered: morphometric data and functional aspects," Journal of Anatomy, vol. 221, no. 1, pp. 1-8, 2012.

[5] K. Chen, Q. Liu, G. Liao et al., "The sound suppression characteristics of wing feather of owl (Bubo bubo)," Journal of Bionic Engineering, vol. 9, no. 2, pp. 192-199, 2012.

[6] T. Bachmann and H. Wagner, "The three-dimensional shape of serrations at barn owl wings: towards a typical natural serration as a role model for biomimetic applications," Journal of Anatomy, vol. 219, no. 2, pp. 192-202, 2011.

[7] T. Geyer, E. Sarradj, and C. Fritzsche, "Measurement of the noise generation at the trailing edge of porous airfoils," Experiments in Fluids, vol. 48, no. 2, pp. 291-308, 2010.

[8] T. Geyer, E. Sarradj, and C. Fritzsche, "Porous airfoils: noise reduction and boundary layer effects," International Journal of Aeroacoustics, vol. 9, pp. 787-820, 2010.
[9] T. Geyer, E. Sarradj, and C. Fritzsche, "Silent owl flight: comparative acoustic wind tunnel measurements on prepared wings," Acta Acustica United with Acustica, vol. 99, no. 1, pp. 139-153, 2013.

[10] M. Herr, "Design criteria for low-noise trailing-edges," in Proceedings of the 13th AIAA/CEAS Aeroacoustics Conference, 2007.

[11] M. S. Howe, "Noise produced by a sawtooth trailing edge," Journal of the Acoustical Society of America, vol. 90, no. 1, pp. 482-487, 1991.

[12] G. M. Lilley, "A study of the silent flight of the owl," AIAA paper, vol. 2340, pp. 1-6, 1998.

[13] G. M. Lilley, "The prediction of air frame noise and comparison with experiment," Journal of Sound and Vibration, vol. 239, no. 4, pp. 849-859, 2001.

[14] T. Bachmann, J. Emmerlich, W. Baumgartner, J. M. Schneider, and H. Wagner, "Flexural stiffness of feather shafts: geometry rules over material properties," Journal of Experimental Biology, vol. 215, no. 3, pp. 405-415, 2012.

[15] A. Ennos, J. Hickson, and A. Roberts, "Functional morphology of the vanes of the flight feathers of the pigeon Columba livia," The Journal of Experimental Biology, vol. 198, pp. 1219-1228, 1995.

[16] J. J. Videler, Avian Flight, Oxford University Press, New York, NY, USA, 2005.

[17] M. Azoulay, A. Veprik, V. Babitsky, and N. Halliwell, "Distributed absorber for noise and vibration control," Shock and Vibration, vol. 18, no. 1-2, pp. 181-219, 2011.

[18] M. R. Mofakhami, H. H. Toudeshky, and S. H. Hashemi, "Noise reduction evaluation of multi-layered viscoelastic infinite cylinder under acoustical wave excitation," Shock and Vibration, vol. 15 , no. 5, pp. 551-572, 2008.

[19] S. M. Hasheminejad and N. Safari, "Dynamic viscoelastic effects on sound wave diffraction by spherical and cylindrical shells submerged in and filled with viscous compressible fluids," Shock and Vibration, vol. 10, no. 5-6, pp. 339-363, 2003.

[20] L. Ren, S. Sun, and C. Xu, "Noise reduction mechanism of nonsmooth leading edge of owl wing," Journal of Jilin University, vol. 38, pp. 126-131, 2008.

[21] H. D. Gruschka, I. U. Borchers, and J. G. Coble, "Aerodynamic noise produced by a gliding Owl," Nature, vol. 233, no. 5319, pp. 409-411, 1971.

[22] Z. Lei, R. Bai, L. Deng, and W. Qiu, "Noncontact optical measurement of CTOA and CTOD for interface crack in DCB test," Optics and Lasers in Engineering, vol. 50, no. 7, pp. 964970, 2012.

[23] W. R. Corning and A. A. Biewener, "In vivo strains in pigeon flight feather shafts: implications for structural design," Journal of Experimental Biology, vol. 201, no. 22, pp. 3057-3065, 1998.

[24] R. H. C. Bonser, "The mechanical properties of feather keratin," Journal of Zoology, vol. 239, no. 3, pp. 477-484, 1996. 
[25] P. Purslow and J. Vincent, "Mechanical properties of primary feathers from the pigeon," The Journal of Experimental Biology, vol. 72, pp. 251-260, 1978.

[26] J. D. Achenbach and C. C. Chao, "A three-parameter viscoelastic model particularly suited for dynamic problems," Journal of the Mechanics and Physics of Solids, vol. 10, no. 3, pp. 245-252, 1962.

[27] S. E. Worcester, "The scaling of the size and stiffness of primary flight feathers," Journal of Zoology, vol. 239, no. 3, pp. 609-624, 1996. 

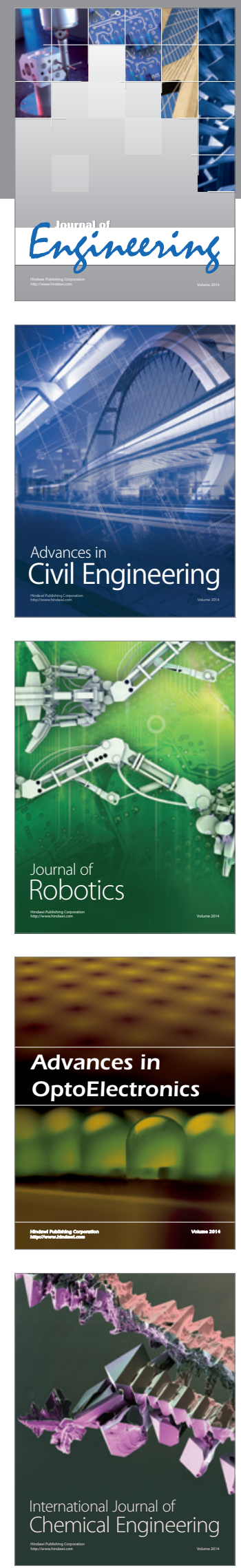

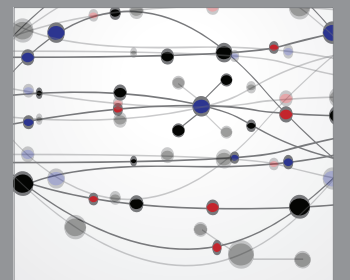

The Scientific World Journal
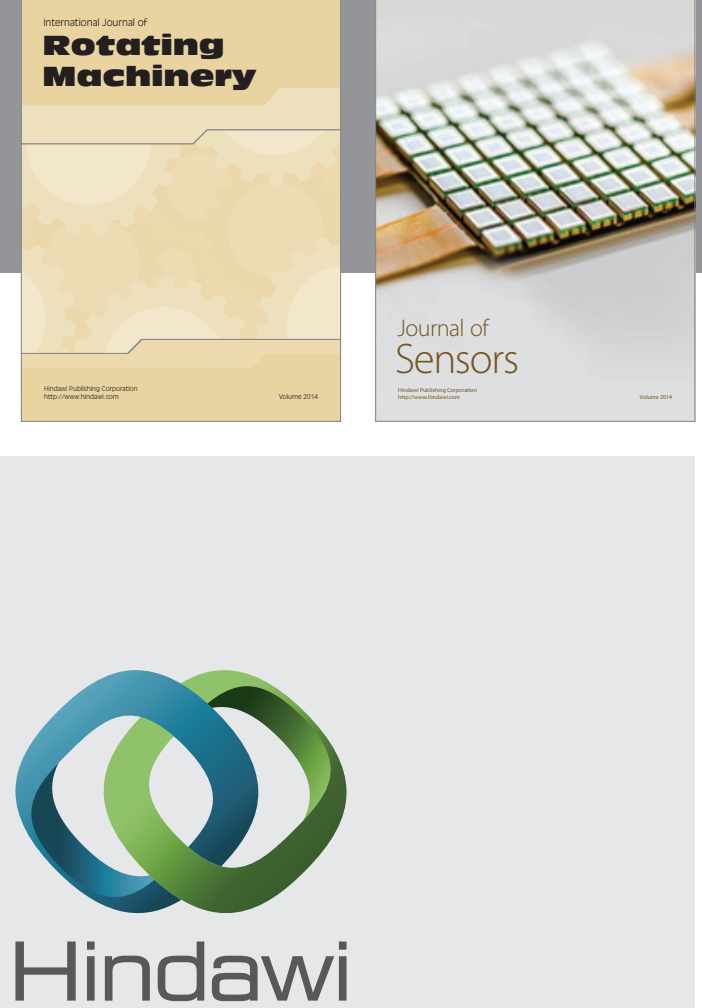

Submit your manuscripts at http://www.hindawi.com
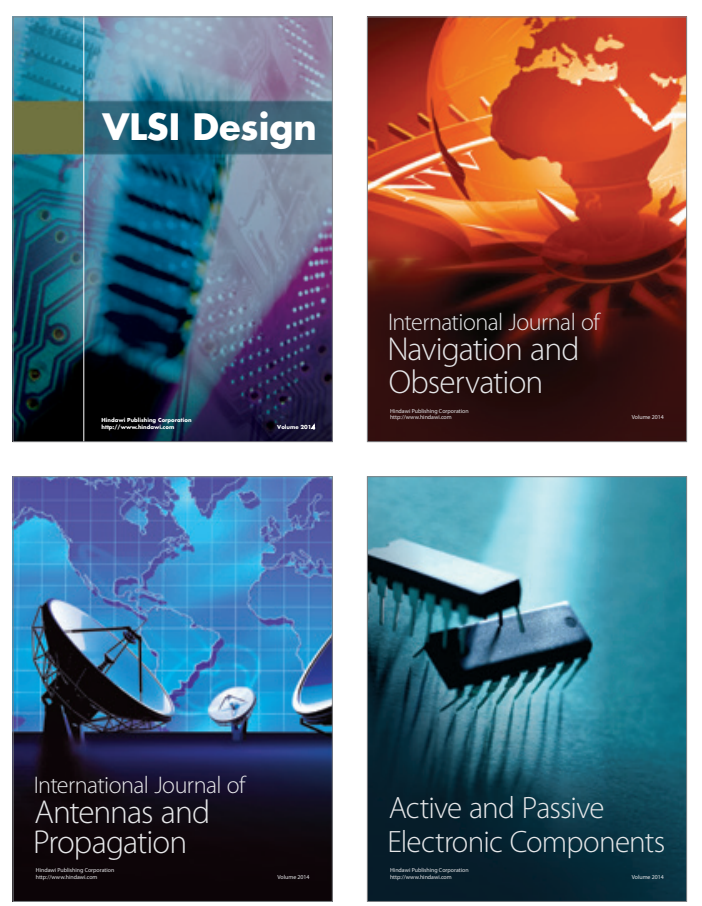
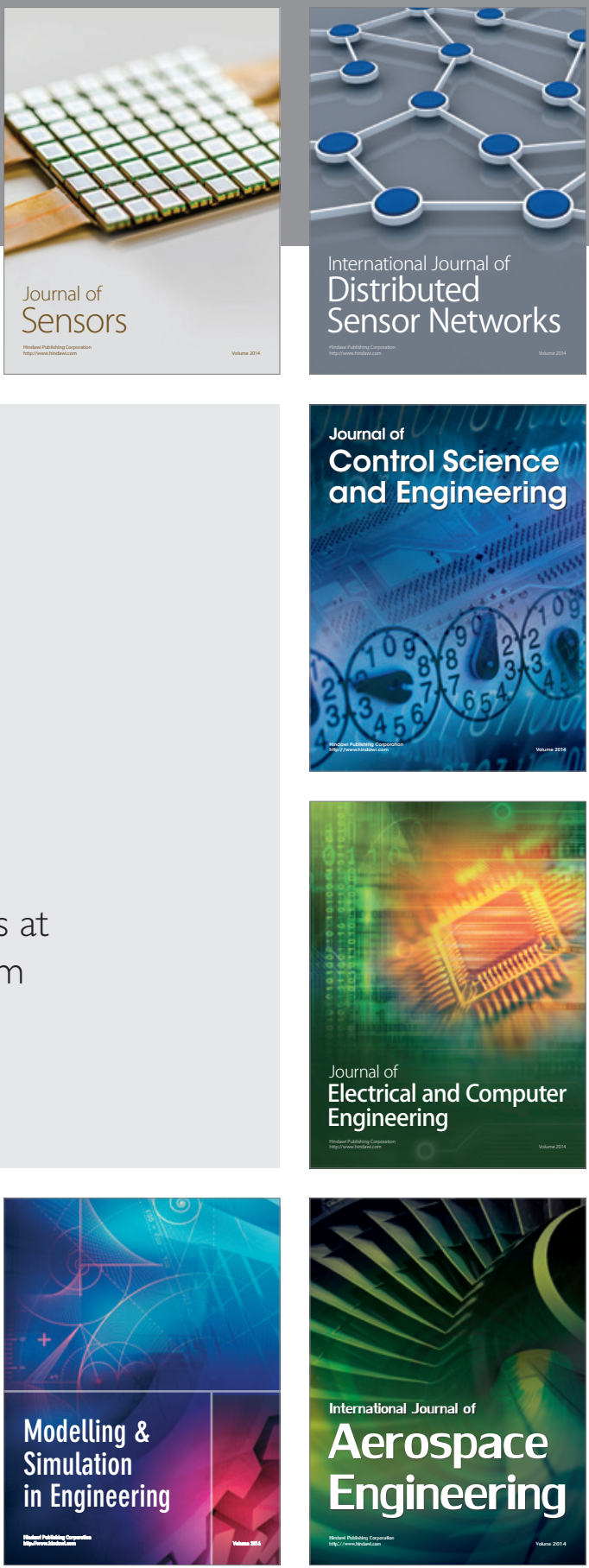

Journal of

Control Science

and Engineering
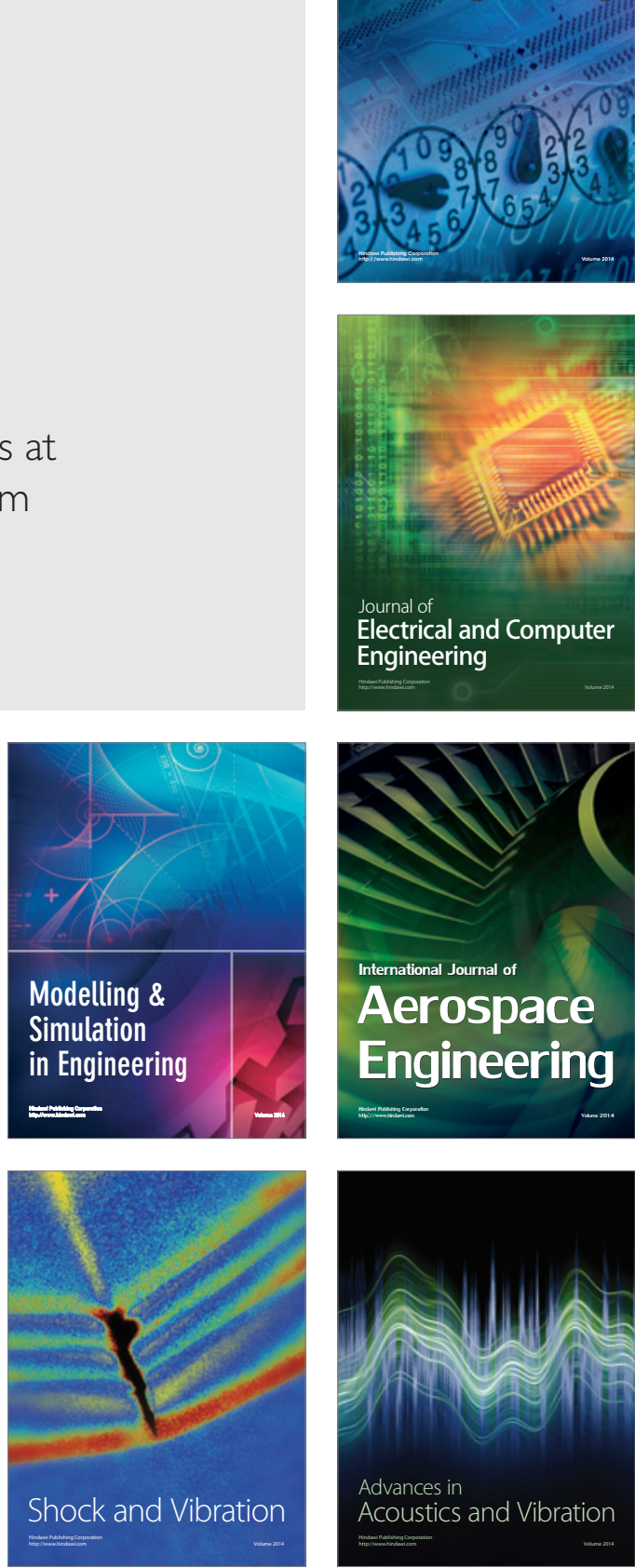